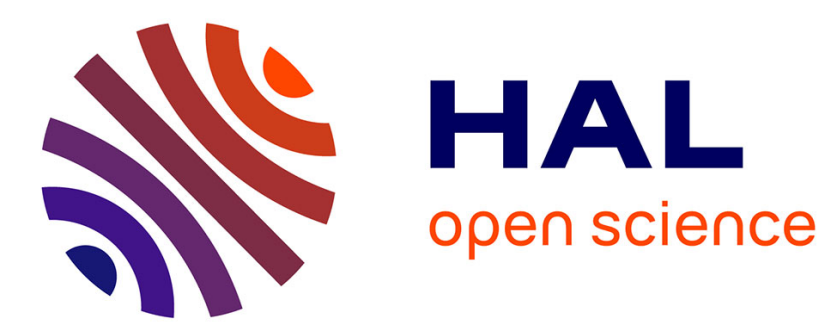

\title{
Adaptive Triangular-Quadrilateral Mesh Generation
}

Houman Borouchaki, Pascal Frey

\section{To cite this version:}

Houman Borouchaki, Pascal Frey. Adaptive Triangular-Quadrilateral Mesh Generation. [Research Report] RR-2960, INRIA. 1996. inria-00073738

\section{HAL Id: inria-00073738 https://hal.inria.fr/inria-00073738}

Submitted on 24 May 2006

HAL is a multi-disciplinary open access archive for the deposit and dissemination of scientific research documents, whether they are published or not. The documents may come from teaching and research institutions in France or abroad, or from public or private research centers.
L'archive ouverte pluridisciplinaire HAL, est destinée au dépôt et à la diffusion de documents scientifiques de niveau recherche, publiés ou non, émanant des établissements d'enseignement et de recherche français ou étrangers, des laboratoires publics ou privés. 


\section{Adaptive Triangular-Quadrilateral Mesh Generation}

Houman BOROUCHAKI and Pascal J. FREY

$$
\mathbf{N}^{\circ} \mathbf{2 9 6 0}
$$

August 20, 1996

THÈME 4 



\title{
RIN RIA
}

\section{Adaptive Triangular-Quadrilateral Mesh Generation}

\section{Houman BOROUCHAKI* and Pascal J. FREY ${ }^{\dagger}$}

Thème 4 - Simulation et optimisation

de systèmes complexes

Projet Gamma

Rapport de recherche $\mathrm{n}^{\circ} 2960$ - August 20, 1996 - 31 pages

\begin{abstract}
In this paper, we begin by recalling an adaptive mesh generation method governed by isotropic and anisotropic discrete metric maps, by means of the generation of a unit mesh with respect to a riemannian structure. We propose then an automatic triangular to quadrilateral mesh conversion scheme, which generalizes the standard case to the anisotropic context. In addition, we introduce an optimal vertex smoothing procedure. Application test examples, in particular a CFD test, are given to demonstrate the efficiency of the proposed method.
\end{abstract}

Key-words: Delaunay triangulation, Anisotropic mesh generation, Adaptive meshing, Quadrilateral mesh, Riemannian structure.

(Résumé : tsvp)

* E-mail : Houman.Borouchaki@inria.fr

$\dagger$ E-mail : Pascal.Frey@inria.fr 


\section{Génération de maillages adaptatifs triangulaires-quadrangulaires}

Résumé : Dans ce rapport, nous rappelons, dans un premier temps, une méthode de génération de maillages adaptatifs gouvernée par une carte discrète de métriques (isotropes ou anisotropes), basée sur la génération de maillages unité par rapport à une structure riemannienne. Nous proposons ensuite un schéma de conversion automatique des triangles en quadrangles, généralisant le cas classique dans un contexte anisotrope. De plus, nous décrivons une procédure de bougé de points optimal. Quelques exemples d'applications, en particulier en mécanique des fluides viennent illustrer l'efficacité de la méthode.

Mots-clé : Triangulation de Delaunay, maillage anisotrope, maillage adaptatif, maillage en quadrangles, structure riemannienne. 


\section{Contents}

1 Introduction 4

2 Unit triangular mesh 6

2.1 Unit mesh of the boundary . . . . . . . . . . . . . 7

2.2 Unit mesh of the domain . . . . . . . . . . . . . 7

3 Adaption scheme $\quad 9$

4 Conversion to unit quadrilateral mesh $\quad \mathbf{1 0}$

4.1 Preliminary definitions . . . . . . . . . . . . . . 11

4.1.1 Scalar product with respect to a metric . . . . . . . . 11

4.1.2 Angle measure with respect to a metric . . . . . . . . . 11

4.1.3 Quality of a quadrilateral element . . . . . . . . . 11

4.2 Scheme of the method . . . . . . . . . . . . . . . 12

4.2.1 Triangle merging procedure . . . . . . . . . . . 12

4.2.2 Conversion of island triangles to quadrilaterals . . . . . . 14

5 Mesh optimization using vertex smoothing 15

6 Numerical results $\quad \mathbf{1 6}$

6.1 Academic test examples ... . . . . . . . . . . 17

6.1.1 Example 1.................. 17

6.1.2 Example 2 . . . . . . . . . . . . . . 17

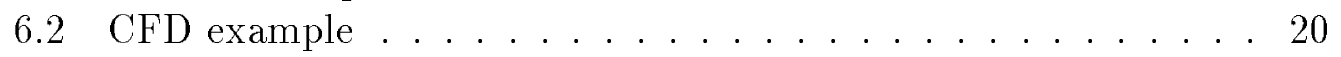

7 Conclusions $\quad 29$

$\operatorname{RR} n^{\circ} 2960$ 
H.Borouchaki and P.J.Frey

\section{Introduction}

Mesh adaption offers the advantages of improving the accuracy of the computed solution in the context of the finite element methods and reducing the number of elements required to capture the behavior of the underlying physical phenomenon $[13,14,16,21]$. This procedure leads to refine or derefine the mesh according to the variation of the gradient of the solution. This information is translated into a size map associated with the initial mesh vertices, which indicates at each vertex the desired size of its surrounding elements. An extension of the latter indicates also the desired sizes not defined in the usual metric but defined in a general riemannian metric making thus the treatment of anisotropic cases possible. The problem we face is to construct a triangular or quadrilateral mesh which respects a given riemannian metric map.

Basically, two different approaches can be envisaged to address the adaptive triangular mesh generation problem of a given domain:

- mesh optimization using refinement and derefinement tools [2, 18, 8] and

- mesh reconstruction of the whole domain [4].

For both cases, numerous algorithms have been proposed which appear to give satisfactory results, in particular when anisotropic specifications are required.

There exists mainly two approaches (direct and indirect) concerning the generation of quadrilateral meshes for domains of arbitrary shape. We briefly review these two classes of methods.

Direct approaches. Among the direct approaches, essentially two methods have been proposed, based on

- the domain decomposition followed by quadrilateral sub-domain filling by means of an algebraic method $[1,19]$ and

- quadrilateral paving techniques [3].

The first method is domain decomposition sensitive and relies on the quasiconvex nature of the resulting sub-domains. The domain decomposition algorithms usually require the local or global knowledge of the domain. In

INRIA 
particular in this last case, the skeleton fully defines and allows an accurate decomposition of the domain. The skeleton extraction of a domain of $I R^{2}$ is an almost solved problem. The second method consists in paving the domain from the boundary to the interior and managing the front collisions. By nature, the performances of this method are closely related to the boundary discretization.

In the case where a constant isotropic metric field is specified, these two classes of methods are likely to lead to the same results. Actually, when using the second method, the advancing-front shock approaches the skeleton. On the other hand, if a generalized metric map is specified, the second method is more likely to respect the field. An improvement of the first method might be to redefine the skeleton with respect to the specified metric map, which is equivalent to supply the domain with a riemannian structure. This latter is a theoretically difficult problem.

Indirect approaches. Given a triangular mesh of the domain, this approach aims at combining triangles into quadrilaterals $[9,10,11,12,17,22]$ and leads to two related merging processes. The triangle merging procedure

1. is driven by the quadrilateral quality and may lead to mixed triangularquadrilateral meshes, or

2. starts from the boundary and moves to the interior of the domain, ensuring an even number of vertices when two fronts collide and results in pure quadrilateral meshes (if the boundary discretization has an even number of vertices).

The first method is conceptually very simple, whereas the second method requires a topological classification of the front collisions and eventually adds vertices to the initial mesh.

We propose an indirect-based method of the first approach. The main contribution of this paper is to extend the triangle merging procedure to the case where a generalized metric map is specified. In addition, we introduce a new mesh optimization technique based on vertex smoothing. Section 2, we recall the unit triangular mesh concept in order to construct the triangular mesh governed by a generalized metric map. The adaption scheme is given

$\operatorname{RR} \mathrm{n}^{\circ} 2960$ 
in section 3. In section 4, we describe the triangular to quadrilateral mesh conversion algorithm. Mesh optimization using vertex smoothing is detailed in section 5. Some numerical results, academic tests and a CFD example, are discussed in section 6 and future works and extension are given in the last section.

\section{Unit triangular mesh}

Let $\Omega$ be a domain of $I R^{2}$, supplied with a riemannian structure. We will discuss the problem of meshing $\Omega$ in such a way that every edge in the resulting mesh is of unit length size. This mesh is called the unit mesh of the domain $\Omega$. The riemannian structure is used to govern the mesh generation process. In practice, this structure is defined, using an interpolation, from a discrete field of metrics associated with the vertices of a given mesh of $\Omega$ (a background mesh). The metric at a mesh vertex $P$ is defined by a symmetric positive definite $2 \times 2$ matrix, denoted as $\mathcal{M}_{2}$ and given by:

$$
\mathcal{M}_{2}(P)=\left(\begin{array}{ll}
a(P) & b(P) \\
b(P) & c(P)
\end{array}\right)
$$

where $a(P)>0$ and $a(P) c(P)-b^{2}(P)>0$. The aim is to obtain a unit mesh with respect to the field $\mathcal{M}_{2}$, i.e. such that every mesh edge $[P X]$ connected to $P$ satisfies at best the relationship (cf. figure 1):

$$
{ }^{t} \vec{P} \vec{X} \mathcal{M}_{2}(P) \vec{P} \vec{X}=1 .
$$

The structure defined by the background mesh and the discrete field of metrics is called the control space. The meshing process of $\Omega$, with respect to the control space, includes the following two stages:

1. the generation of the unit mesh of the boundary of $\Omega$ and

2. the generation of the unit mesh of $\Omega$ using the unit mesh of its boundary as input.

Afterwards, we briefly recall the main features of the unit meshing algorithm and refer the reader to $[4,5]$ for more details.

INRIA 


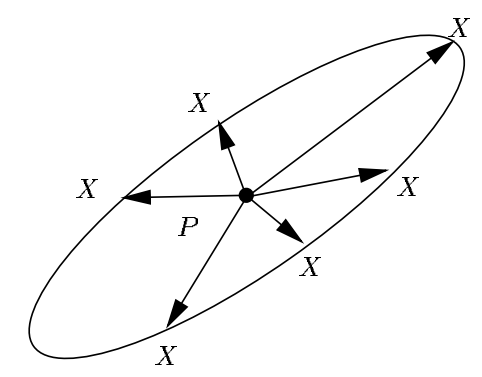

Figure 1: Unit circle, anisotropic case.

\subsection{Unit mesh of the boundary}

We assume that a mathematical model defines the geometry of the domain. For example, this model can be derived from a sufficiently fine initial boundary discretization. Thus, a unit mesh of the boundary with respect to a given riemannian structure is a discretization of this model, obtained by subdividing it with unit arc length segments with respect to the control space. To this end, we can approximate the model with a polygonal segment, which is later subdivided into unit length segments.

\subsection{Unit mesh of the domain}

The unit mesh of the boundary of $\Omega$ provides a set of constrained edges having as endpoints a set of points, denoted as $\mathcal{S}(\Omega)$. At first, an initial constrained mesh of $\Omega$ is generated, whose sole vertices are the members of $\mathcal{S}(\Omega)$, respecting the constrained edges. A new mesh is then obtained by adding iteratively field points inside this mesh, and optimized so that as to obtain the unit mesh of the domain. The mesh is initialized by the initial constrained mesh. At each iteration, the internal mesh edges are analyzed and the internal field points

- are generated along the edges such as to subdivide them into unit length segments (new points must not be too close to the existing vertices) and

- are inserted into the current mesh via the constrained Delaunay kernel applied in a riemannian context.

$\operatorname{RR} \mathrm{n}^{\circ} 2960$ 
This process is repeated as long as the current mesh is modified. Afterwards, we review the generalized constrained Delaunay kernel, the length of a segment computation and the optimization procedure.

Generalized constrained Delaunay kernel. The classical constrained Delaunay kernel is a procedure resulting in the insertion of one internal point in a (Delaunay) triangulation, based on a proximity criterion. Formally speaking, the constrained Delaunay kernel can be written as [20]:

$$
T=T-C(P)+B(P)
$$

where $C(P)$ is the cavity associated with point $P$ and $B(P)$ is the triangulation of $C(P)$ enclosing $P$ as a vertex, $T$ denoting the current Delaunay mesh. The cavity is constructed using a proximity criterion, written as:

$$
\{K, K \in T, P \in \operatorname{Disc}(K) \text { and } P \text { visible from any vertex of } K\}
$$

where $\operatorname{Disc}(K)$ is the circumdisc of $K$.

The generalization of this procedure consists in redefining the cavity $C(P)$ in a riemannian context [6]. Therefore, we define first the Delaunay measure $\alpha_{\mathcal{M}_{2}}$ associated with the couple $(P, K)$, with respect to a given metric $\mathcal{M}_{2}$ :

$$
\alpha_{\mathcal{M}_{2}}(P, K)=\left[\frac{d\left(O_{K}, P\right)}{r_{K}}\right]_{\mathcal{M}_{2}},
$$

where $O_{K}$ (resp. $r_{K}$ ) is the center (resp. radius) of the circumdisc of $K$ and $[*]_{\mathcal{M}_{2}}$ indicates that the quantity $*$ is evaluated in the euclidean space characterized by the metric $\mathcal{M}_{2}$. The usual proximity criterion, $P \in \operatorname{Disc}(K)$, is expressed as $\alpha_{\mathcal{I}_{2}}(P, K)<1$, where $\mathcal{I}_{2}$ is the identity metric. The cavity $C(P)$ is then redefined as:

$$
C(P)=C_{1}(P) \cup C_{2}(P)
$$

with

$$
C_{1}(P)=\{K, K \in T, K \text { including } P\}
$$


and

$$
\begin{gathered}
C_{2}(P)=\left\{K, K \in T, \exists K^{\prime} \in C(P), K \text { adjacent to } K^{\prime},\right. \\
\alpha_{\mathcal{M}(P)}(P, K) \prod_{V} \alpha_{\mathcal{M}(V)}(P, K)<1, \mathrm{~V} \text { vertex of } K, \\
P \text { visible from the vertices of } K\} .
\end{gathered}
$$

Hence, the $C(P)$ is constructed by adjacency from the elements of $C_{1}(P)$. With this definition, we can deduce that the generalized cavity is star-shaped with respect to $P$ and the triangulation of $B(P)$ is then valid.

Length of a segment. The length of a segment $[P Q]=(P+t \overrightarrow{P Q})_{0 \leq t \leq 1}$ of $\Omega$ is given by:

$$
l([P Q])=\int_{0}^{1} \sqrt{{ }^{t} \overrightarrow{P Q} \mathcal{M}_{2}(P+t \overrightarrow{P Q}) \overrightarrow{P Q}} d t
$$

where $\mathcal{M}_{2}(P+t \overrightarrow{P Q})$ is the metric at point $P+t \overrightarrow{P Q}$ of the segment $[P Q]$, this metric being well defined through the control space.

Optimization. This procedure aims at improving the mesh edge lengths, by means of:

- edge swapping, if the alternative edges are closer to the unit length and

- optimal vertex smoothing, which consists in moving $P$, "step-by-step", to a position where all incident edges are close to the unit length size.

\section{Adaption scheme}

If the control space, consisting of the initial mesh and the relative discrete metric map, does not match exactly the ideal continuous field of metrics, the discrete map is enriched to govern the meshing process more accurately. The whole procedure is then rerun. Indeed, the unit mesh generation scheme can be easily extended to construct a loop of adaption. To this end, the control space at each iteration $i$ is defined by the mesh at iteration $i-1$ and a metric

RR n ${ }^{\circ} 2960$ 
map specified at all vertices of this mesh. The adaption scheme is given as follows:

- Data of $F_{\text {geom }}$, the initial discretization of the boundary of $\Omega$ serving as definition for the geometry and the model Model $_{\text {geom }}$.

- Initial discretization $F_{0}$ of Model $_{\text {geom }}$ according to a size map $H_{0}$.

- Construction of the initial mesh $T_{0}$ using $F_{0}$ and $H_{0}$ as data.

- Adaption loop (starting at $i=1$ )

- data of a metric map $H_{i}$ on $T_{i-1}$.

- discretization $F_{i}$ of model Model $_{\text {geom }}$ governed by the control space $\left(T_{i-1}, H_{i}\right)$.

- adapted mesh $T_{i}$ using $F_{i}$ and the control space $\left(T_{i-1}, H_{i}\right)$.

- iteration $i=i+1$, if required.

The above scheme is iterated until an almost satisfactory mesh $T_{i}$ is obtained with respect to $\left(T_{i}, H_{i+1}\right)$. In other words, the edges in $T_{i}$ have a length almost equal to unity in the control space. In this case, the mesh $T_{i}$ is said to be compatible with the metric maps $H_{i}$ or $H_{i+1}$.

\section{Conversion to unit quadrilateral mesh}

Given a unit mesh and an associated compatible metric map, the main idea of the conversion to unit quadrilateral mesh consists in pairing the triangles to form quadrilateral elements by controlling the quality. Obviously, this wellknown strategy cannot always produce a pure quadrilateral mesh and may lead to a mixed mesh, composed of both triangular and quadrilateral elements. The proposed method generalizes the merging process to the case of a domain supplied with a riemannian structure and tends to create optimal quadrilaterals via the specified structure. Another goal can be to minimize the number of isolated triangles.

Therefore, we introduce several preliminary definitions, prior to define the quadrilateral quality and we propose an algorithm suitable for the quadrilateral meshing process with respect to the given objectives.

INRIA 


\subsection{Preliminary definitions}

We review the notion of the scalar product, the angle measure and the angle quality with respect to a given metric and introduce the "generalized" quality for a quadrilateral.

\subsubsection{Scalar product with respect to a metric}

The scalar product of two given vectors $\vec{u}$ and $\vec{v}$ in the euclidean space characterized by a metric $\mathcal{M}_{2}(X)$ is given by:

$$
[\langle\vec{u}, \vec{v}\rangle]_{\mathcal{M}_{2}(X)}={ }^{t} \vec{u} \mathcal{M}_{2}(X) \vec{v},
$$

and the norm of a vector $\vec{w}$ by:

$$
[\|\vec{w}\|]_{\mathcal{M}_{2}(X)}=\sqrt{t \vec{w} \mathcal{M}_{2}(X) \vec{w}}
$$

\subsubsection{Angle measure with respect to a metric}

Let $A B C$ be three points such that $\overrightarrow{B C} \times \overrightarrow{B A}>0$ where $\times$ denotes the cross product in the usual euclidean metric. The angle measure in radians of the angle between the two vectors $\overrightarrow{B C}$ and $\overrightarrow{B A}$ with respect to a metric $\mathcal{M}_{2}(X)$ is given by:

$$
\left[\theta_{B}\right]_{\mathcal{M}_{2}(X)}=[(\overrightarrow{B C}, \overrightarrow{B A})]_{\mathcal{M}_{2}(X)}=\arccos \left(\frac{[\langle\overrightarrow{B C}, \overrightarrow{B A}\rangle]_{\mathcal{M}_{2}(X)}}{[\|\overrightarrow{B C}\|]_{\mathcal{M}_{2}(X)}[\|\overrightarrow{B A}\|]_{\mathcal{M}_{2}(X)}}\right)
$$

Remark 1 In the case where $\overrightarrow{B C} \times \overrightarrow{B A}<0$ the angle is then given by:

$$
\left[\theta_{B}\right]_{\mathcal{M}_{2}(X)}=2 \pi-[(\overrightarrow{B A}, \overrightarrow{B C})]_{\mathcal{M}_{2}(X)} .
$$

\subsubsection{Quality of a quadrilateral element}

Let $\mathcal{Q}=[A B C D]$ be a quadrilateral element where $A, B, C, D$ are given in counterclockwise order. The quality of $\mathcal{Q}$ can be defined from the measure of

$\operatorname{RR} \mathrm{n}^{\circ} 2960$ 
the angle (in $[0,2 \pi]$ ) qualities of $\mathcal{Q}$. This latter is optimal for an angle value of $\frac{\pi}{2}$ radians and decreases as much as its difference with this value increases. Moreover, the angle quality is equal to 0 for an angle measure in $[\pi, 2 \pi]$. By normalizing the optimal value to 1 and by considering, for instance, a linear variation of the quality, it is possible to define an angle quality function $\phi$ with respect to a given metric $\mathcal{M}_{2}(X)$, as (cf. figure 2):

$$
\phi\left([\theta]_{\mathcal{M}_{2}(X)}\right)= \begin{cases}\frac{2[\theta]_{\mathcal{M}_{2}(X)}}{\pi} & \text { if } 0 \leq[\theta]_{\mathcal{M}_{2}(X)}<\frac{\pi}{2} \\ 2-\frac{2[\theta]_{\mathcal{M}_{2}(X)}}{\pi} & \text { if } \frac{\pi}{2} \leq[\theta]_{\mathcal{M}_{2}(X)}<\pi \\ 0 & \text { if } \pi \leq[\theta]_{\mathcal{M}_{2}(X)}\end{cases}
$$

where $[\theta]_{\mathcal{M}_{2}(X)}, 0 \leq[\theta]_{\mathcal{M}_{2}(X)} \leq 2 \pi$, represents the measure of an angle (in radians) in the metric $\mathcal{M}_{2}(X)$.

The quality $q(\mathcal{Q})$ of $\mathcal{Q}$ is then defined as the minimum value of the angle quality function, computed with respect to the metrics associated to its vertices. Hence, we have:

$$
q(\mathcal{Q})=\min _{(X, Y) \in\{A, B, C, D\}} \phi\left(\left[\theta_{Y}\right]_{\mathcal{M}_{2}(X)}\right) .
$$

Remark 2 The quality of a quadrilateral element in the riemannian case requires the evaluation of 16 quality measures in the euclidean case.

\subsection{Scheme of the method}

It is possible to govern the triangle combination procedure by using the usual approach described hereafter. Isolated triangles may be present in the final mesh. To obtain a pure quadrilateral mesh, we review a standard triangleremoval procedure.

\subsubsection{Triangle merging procedure}

Let $\mathcal{T}$ be the initial unit triangular mesh. Each pair of adjacent triangles is likely to form a quadrilateral element and is identified by the common edge. 


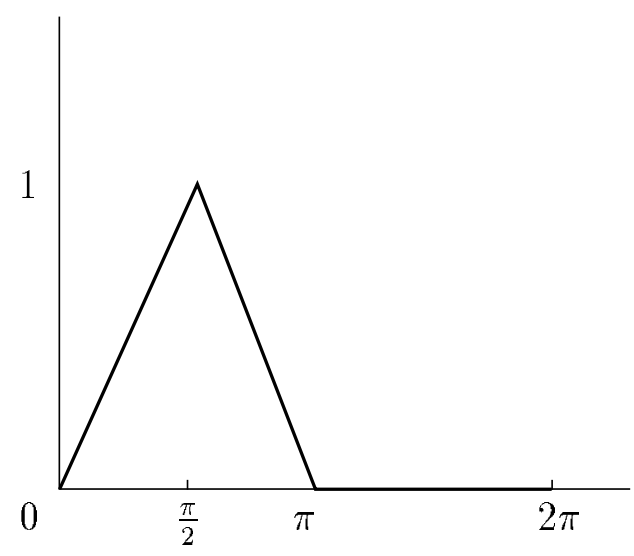

Figure 2: Angle quality.

A simplified measure of the corresponding quadrilateral quality is associated with each edge (cf. figure 2). Let $[A B C]$ and $[A C D]$ be two adjacent triangles, sharing the edge $a=[A C]$. If $\theta_{A}=(\overrightarrow{A B}, \overrightarrow{A D})$ and $\theta_{C}=(\overrightarrow{C D}, \overrightarrow{C B})$, the quality of $a$ can then be defined as (cf. figure 3 ):

$$
q(a)=\min \left(\left[\theta_{A}\right]_{\mathcal{M}_{2}(A)},\left[\theta_{A}\right]_{\mathcal{M}_{2}(C)},\left[\theta_{C}\right]_{\mathcal{M}_{2}(A)},\left[\theta_{C}\right]_{\mathcal{M}_{2}(C)}\right) .
$$

The edge list of $\mathcal{T}$ is sorted according in the decreasing order according to the quality, the corresponding quadrilaterals are constructed and their edges are removed from the list. The merging process is thus governed by the quality measure, even if the number of isolated triangles is not minimized. It is possible to minimize the number of triangles by governing the combination of triangles by adjacency, from the existing quadrilaterals. To this end, it is sufficient to redefine the edge quality $q^{*}(a)$ of edge $a$ as:

$$
q^{*}(a)=q(a)-q([A B])-q([B C])-q([C D])-q([D A])
$$

Remark 3 With this new definition, the edge list must be updated after each quadrilateral creation. This operation can be efficiently implemented using a heap structure. In addition, two parameters, $q_{t}(=0.1)$ and $q_{d}(=-0.5)$, are used in order to control the triangles merging:

$\operatorname{RR} \mathrm{n}^{\circ} 2960$ 


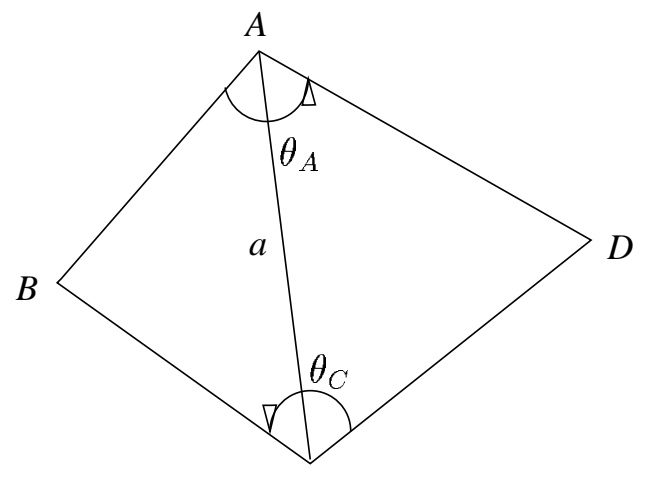

C

Figure 3: Edge quality.

- if $q(a)<q_{t}$ then the edge a is not taken into account,

- if the edge a is a boundary edge or if it is an edge of a newly created quadrilateral then set $q^{*}(a)=q_{d}$.

The merging process by adjacency has an undesirable side effect, the mesh quality is globally degraded.

\subsubsection{Conversion of island triangles to quadrilaterals}

Each isolated triangle is split into three quadrilaterals according to the usual scheme, adding three vertices in the middle of the edges and a vertex at the barycenter of the element. To preserve the mesh conformity, a similar process is also applied to all quadrilaterals, thus propagating a uniform refinement.

Remark 4 Mesh refinement has the disadvantage of producing a mesh that is no longer compatible with the specified metric map. It is possible to retrieve the compatibility by governing the initial triangular mesh creation process using the $\left\{\frac{\mathcal{M}_{2}(P)}{4}\right\}$ metric map, where $\left\{\mathcal{M}_{2}(P)\right\}$ is the initial field. In the isotropic case, this is equivalent to multiply the specified vertex size by two. 


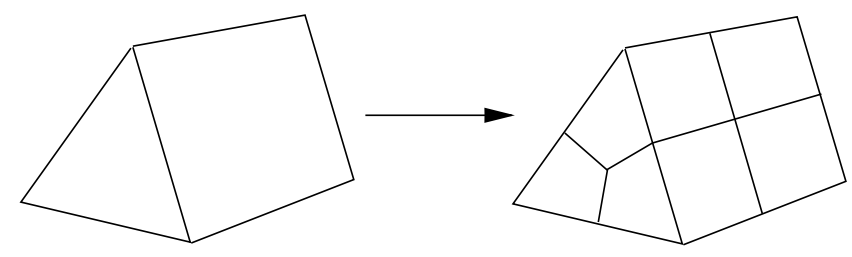

Figure 4: Mesh refinement.

\section{Mesh optimization using vertex smoothing}

This last stage is required because the initial mesh $\mathcal{T}$ is optimal with respect to the triangle quality instead of the quadrilateral quality and because of the introduction of the extra vertices during the conversion of the island triangles. Usually, this step consists in moving the mesh vertices in order to optimize the edge lengths. In the case of a triangular mesh, this scheme leads to a satisfactory result. However, the algorithm cannot be applied to quadrilateral meshes. Actually, a diamond-shaped element is optimal with respect to the edge length criteria. Therefore, we propose a modified technique which consists in using the diagonals of the quadrilaterals.

Let $P$ be a mesh vertex, $n$ (resp. $m$ ) be the number of quadrilaterals $\left\{\left[P P_{i} Q_{i} P_{i+1}\right]\right\}$ (resp. triangles $\left\{\left[P P_{i} P_{i+1}\right]\right\}$ ) sharing $P,\left[P P_{i}\right]$ are the edges and $\left[P Q_{i}\right]$ the diagonals of elements. We define the points $P_{i}^{*}$ and $Q_{i}^{*}$ such that:

- $\overrightarrow{P_{i} P_{i}^{*}}$ is colinear to $\overrightarrow{P_{i} P}$, the length of $\left[P_{i} P_{i}^{*}\right]$ is equal to the unit length (in the control space) and $P_{i}$ and $P_{i}^{*}$ on the same side of $P$. Point $P_{i}^{*}$ is given by the approximate formula:

$$
P_{i}^{*} \approx P_{i}+\frac{\overrightarrow{P_{i} P}}{l\left(\left[P_{i} P\right]\right)}
$$

- $\overrightarrow{Q_{i} Q_{i}^{*}}$ is colinear to $\overrightarrow{Q_{i} P}$, the length of the segment $\left[Q_{i} Q_{i}^{*}\right]$ is equal to $\sqrt{2}$ and $Q_{i}$ and $Q_{i}^{*}$ on the same side of $P$. Point $Q_{i}^{*}$ is given by the approximate formula:

$$
Q_{i}^{*} \approx Q_{i}+\sqrt{2} \frac{\overrightarrow{Q_{i} P}}{l\left(\left[Q_{i} P\right]\right)}
$$

RR $n^{\circ} 2960$ 


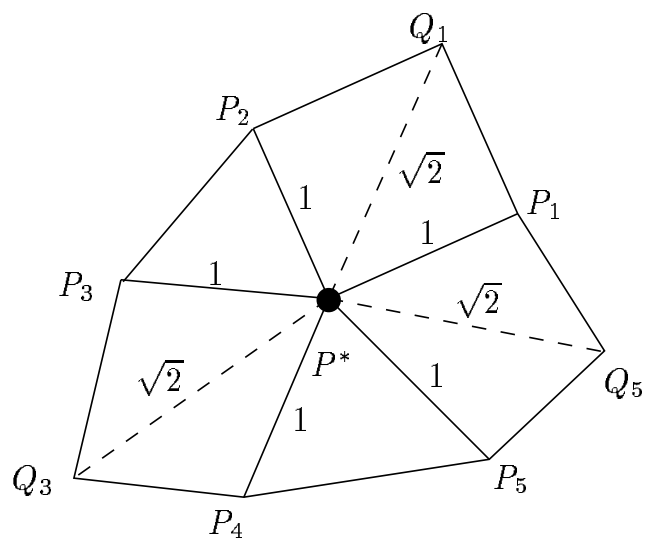

Figure 5: Optimal position of pointP.

The smoothing procedure consists in moving the point $P$ step-by-step toward the point $P^{*}$, barycenter of the points $P_{i}^{*}$ and $Q_{i}^{*}$, if the quality of the worst element of $\left\{\left[P P_{i} Q_{i} P_{i+1}\right]\right\}$ is improved. The new position of $P$ is given by:

$$
P+\omega \overrightarrow{P P^{*}}
$$

where $\omega$ is a relaxation parameter.

Remark 5 The length of each segment is evaluated via the integral of the equation 9. It is sufficient to apply a Simpson formula with three points (the two endpoints and the middle point) to evaluate the integral.

If the final mesh is not satisfactory with respect to the normalized edge lengths, additional quality optimization procedures based on local topological modifications can be used [10].

\section{$6 \quad$ Numerical results}

In this section, two class of examples are given, concerning two academic tests and a CFD problem. In the academic tests, the metric map used to govern the mesh generation is user-specified. In the CFD example, the metric map is given by an a posteriori error estimate based on the hessian of the solution.

INRIA 


\subsection{Academic test examples}

Two academic test examples are given.

\subsubsection{Example 1}

In the first test (by Lewis et al. [11]), the domain is the area of a rectangle defined by

$$
\begin{array}{r}
-1.25 \leq x \leq 1.25 \\
-0.5 \leq y \leq 1.25
\end{array}
$$

supplied with an isotropic metric map indicating at each $(x, y)$ of the domain the desired sizes $h(x, y)$ :

$$
h(x, y)=0.01[1+f(x, y)]
$$

where

$$
f(x, y)=30\left(y-x^{2}\right)^{2}+(1-x)^{2} .
$$

The unit mesh with respect to the isotropic map is shown figure 6 (top). This mesh contains 3500 vertices and 6933 triangles. The normalized edge lengths range from 0.57 to 1.56 , with $95 \%$ between 0.75 and 1.33 . The figure 6 (bottom) shows the mixed mesh after the triangle merging which contains 3417 (97\%) quadrilaterals and $99(3 \%)$ triangles. The corresponding mean quality is 0.63 . The figure 7 (top) illustrates the mixed mesh after the refinement procedure. The final mesh after optimization, figure 7 (bottom), contains 14023 vertices and 13965 quadrilaterals, the worst quality is 0.26 (2 elements) and the mean quality is $0.75,91 \%$ of the vertices are of degree 4 and the minimal and maximal values are 3 and 6 , respectively. The normalized edge lengths of this mesh range from 0.4 ( 9 edges) to 1.7 and $90 \%$ between 0.75 and 1.33 . The whole process has taken 15 seconds of CPU time on a HP735/99Mhz machine.

\subsubsection{Example 2}

In the second test, the domain is the area of a unit square defined by

$$
\begin{aligned}
& 0 \leq x \leq 1 \\
& 0 \leq y \leq 1
\end{aligned}
$$

RR $n^{\circ} 2960$ 


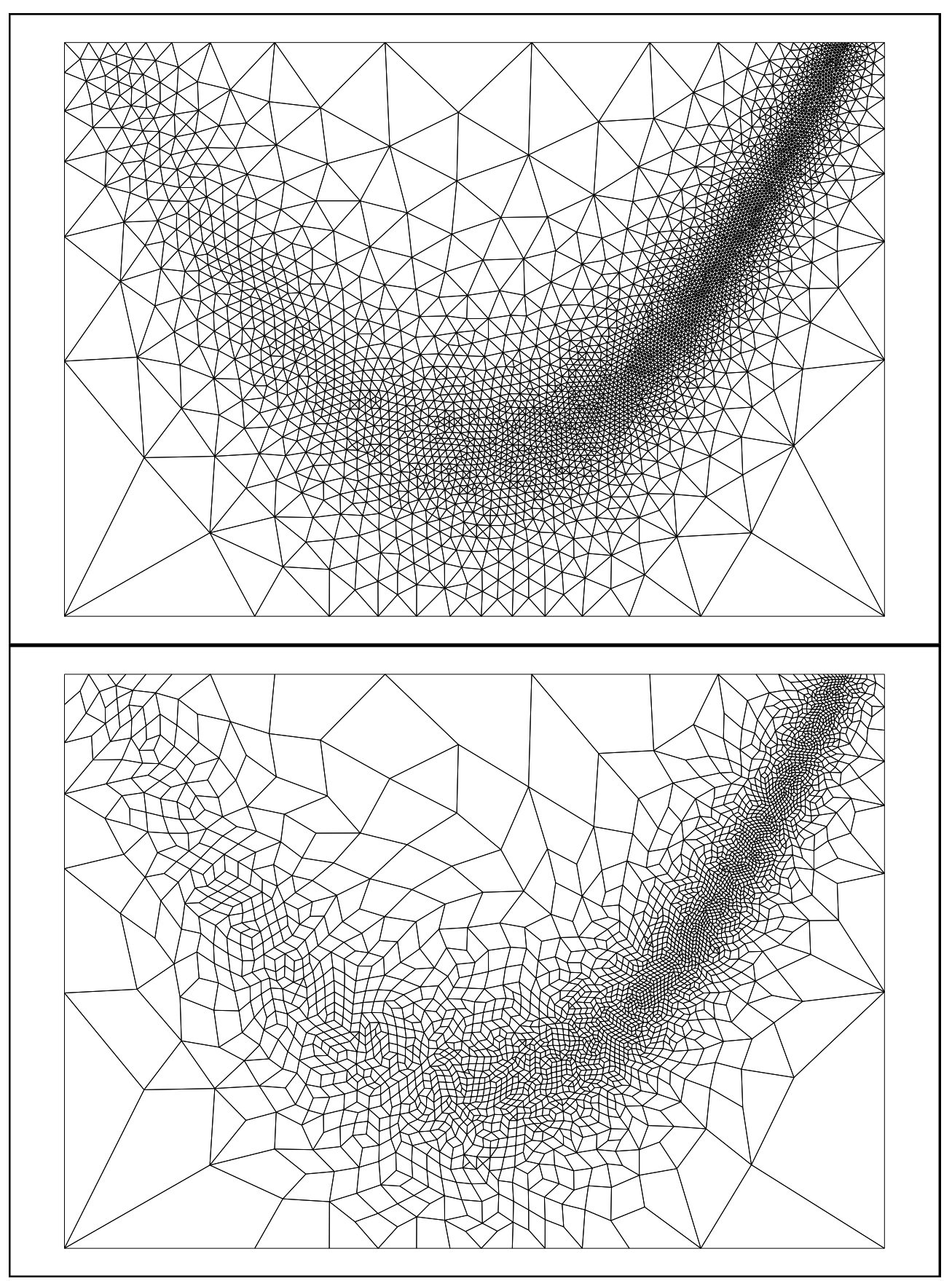

Figure 6: Triangular adaptive mesh and mixed triangular-quadrilateral mesh after triangle merging.

INRIA 


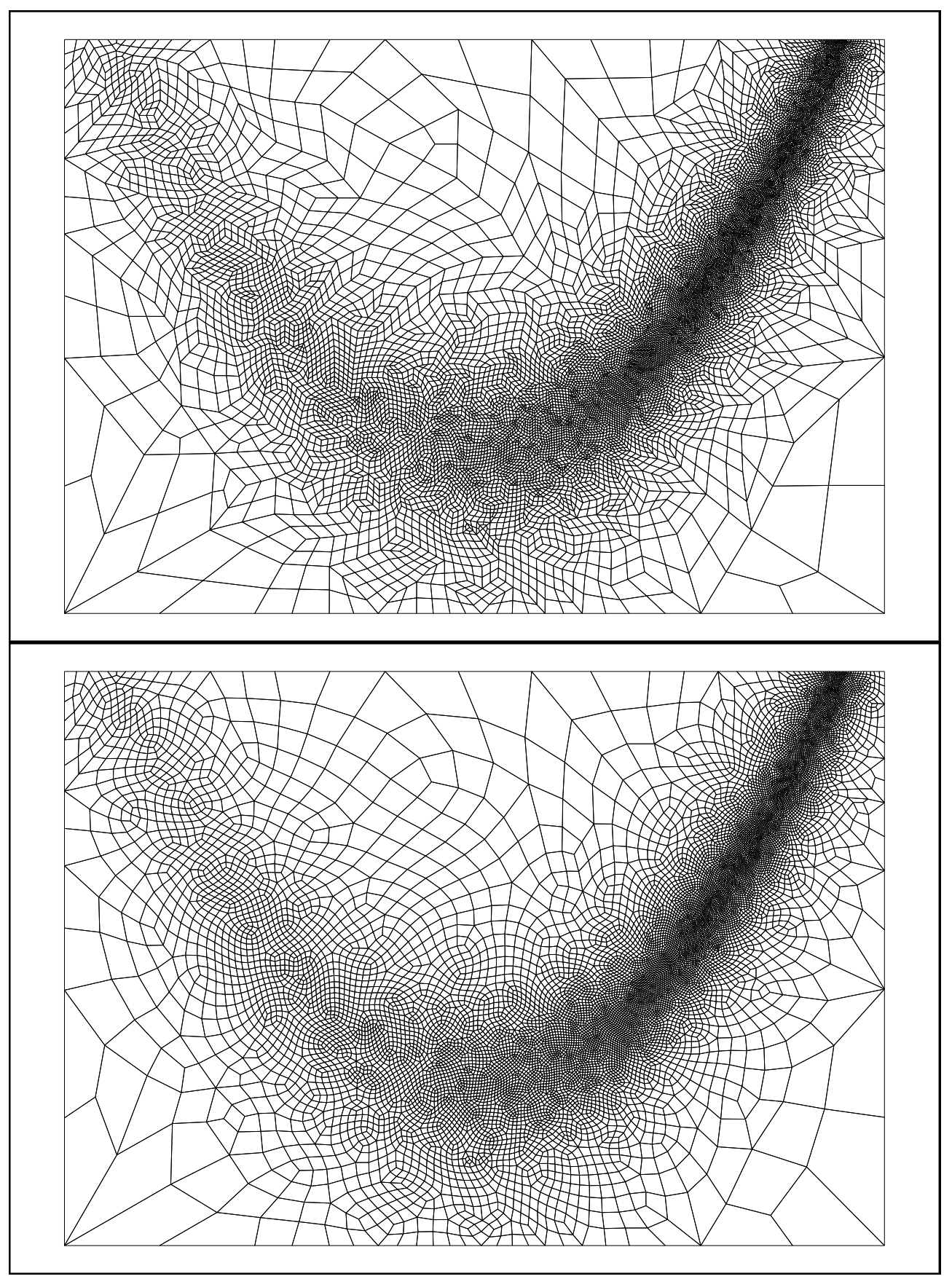

Figure 7: Mixed mesh after refinement and final mesh after optimization.

RR $n^{\circ} 2960$ 
without the area of a disk of radius 0.15 and center $(0.75,0.75)$. We considered two metric maps, isotropic and anisotropic. The isotropic map requires a size of 0.003 along the medial axis of the domain and a linear size grow-up from the medial axis to the interior of the domain. The anisotropic map requires stretched elements along the medial axis and a linear variation of the metrics in order to have isotropic elements from the medial axis to the interior of the domain. The corresponding meshes (as in example 1) for the isotropic map are given in the figure 8. After the merging process, $96 \%$ of the elements are quadrilaterals. The minimal quality of the final mesh is 0.3 and the mean quality is 0.73 . The mesh contains 46438 edges and the corresponding normalized edge lengths range between 0.4 (16 edges) and 2. (7 edges), $87 \%$ between 0.75 and 1.33 . The total speed-up of the quadrilateral mesh generation is 1400 quadrilaterals per seconds.

The meshes for the anisotropic map are given in the figure 9. After the merging process, $97 \%$ of the elements are quadrilaterals. The minimal quality of the final mesh is 0.1 and the mean quality is 0.68 . The mesh contains 25381 edges and the normalized edge lengths range between 0.35 (20 edges) and 2.5 ( 5 edges), $88 \%$ between 0.75 and 1.33 . The stretching factor along the medial axis is about 20 . The speed-up of the quadrilateral mesh generation is 1300 quadrilaterals per seconds.

\subsection{CFD example}

A Mach 2 supersonic flow at Reynolds 10000 is applied to a NACA0012 airfoil. The triangular mesh has been adapted 6 times in the anisotropic context in order to identify the shock, boundary layer and wake regions and even the weak shocks at the trailing edge have been detected. Each mesh adaption has been followed by 500 iterations of a Navier-Stokes triangle-based solver [15]. The quadrilateral mesh has been obtained from the triangular mesh and the associated metric map, to underscore the capabilities of the proposed triangle merging technique. The whole process has taken 3620 seconds of CPU time (HP735/99Mhz), 3600 seconds for the CFD solver and 20 seconds for the meshing procedure.

Figure 10 shows the initial isotropic mesh (iteration 0 ) with 1337 vertices and 2668 triangles, the corresponding quadrilateral mesh with 5238 vertices 


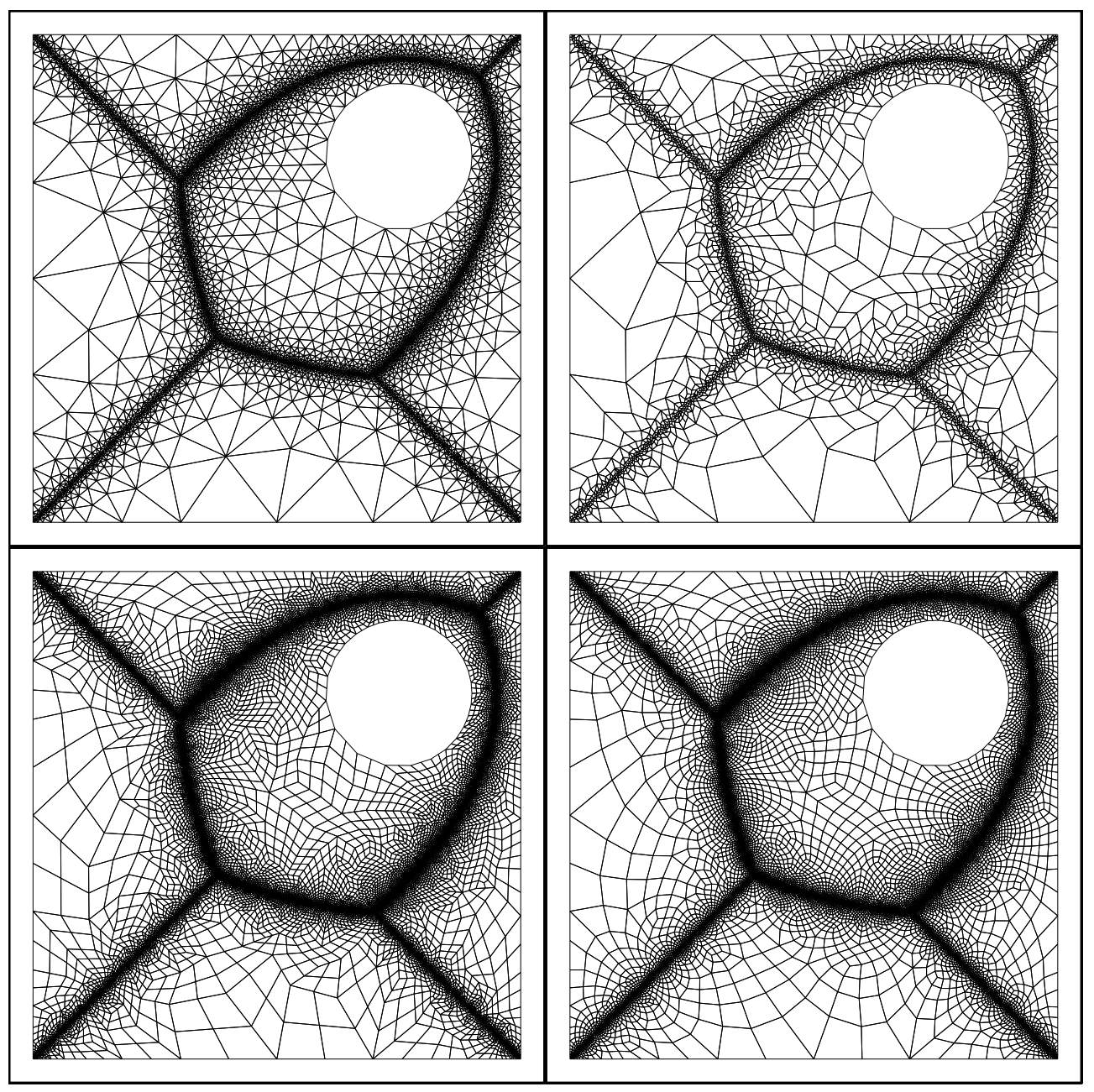

Figure 8: Meshes of example 2, isotropic map.

RR $n^{\circ} 2960$ 


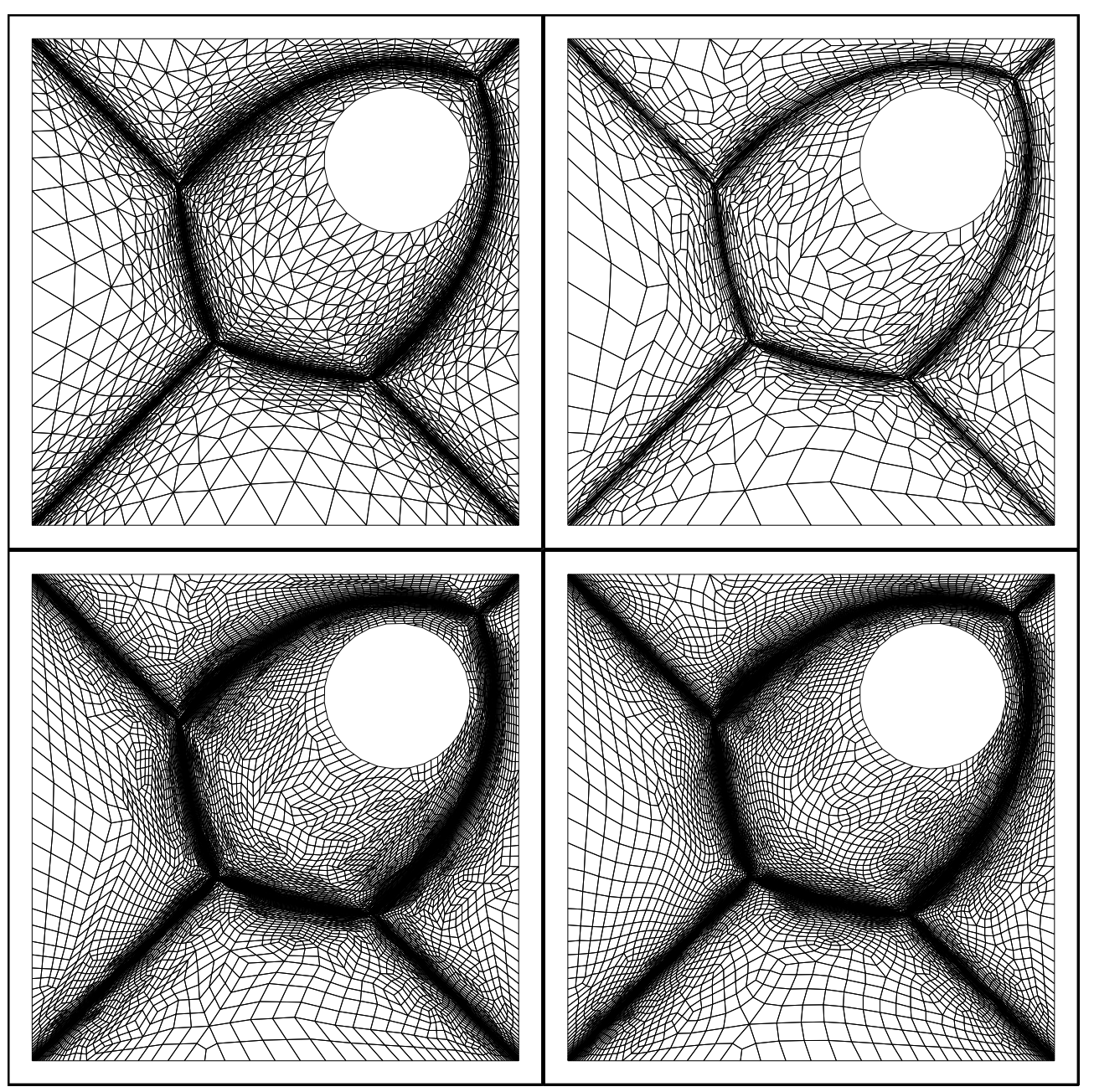

Figure 9: Meshes of example 2, anisotropic map. 
and 5109 quadrilaterals. Similarly, figure 11 shows the final anisotropic mesh (iteration 6 ) with 3846 vertices and 7686 triangles and the corresponding quadrilateral mesh with 15272 vertices and 14897 quadrilaterals, the stretching factor is about 286 .

The metric map related to the final mesh is shown on figures 12 , where at each vertex the associated ellipse represents the desired size in any direction. The reader can refer to [5] for more details about the computation of the anisotropic metric map, derived from an a posteriori error estimate based on second order derivatives of the solution.

The histogram 14 shows the triangular mesh quality as defined in [4] (the quality is a function varying in $[0,1])$. The worst value is 0.1 and $90 \%$ of the elements have a quality better than 0.5. The triangular mesh contains 11151 edges. The histogram 15 shows the normalized edge lengths of the triangular mesh. The minimum value is 0.31 , the maximum value is $9.87,37$ edges have a normalized edge length lesser than 0.5 and 120 have a normalized edge length bigger than 2 . This result can be explained by the discontinuities of the metric map near the shock regions (cf. figure 13).

The histogram 16 shows the quadrilateral mesh quality. The minimum value is 0.05 and the mean quality is 0.64 . The quadrilateral mesh contains 30169 edges. The histogram 17 shows the normalized edge lengths of the quadrilateral mesh. The minimum value is 0.07 , the maximum value is $15.56,578$ edges have a normalized edge length lesser than 0.5 and 241 have a normalized edge length bigger than 2 .

We can appreciate on the figure 18 the quality of the solution after adaptive mesh generation applied to this CFD problem. The figure 18 (top) shows the solution obtained using the fixed initial mesh after 3000 iterations of the Navier-Stokes solver and the figure 18 (bottom) illustrates the iso-density contour lines of the solutions using adaption.

$\operatorname{RR} \mathrm{n}^{\circ} 2960$ 


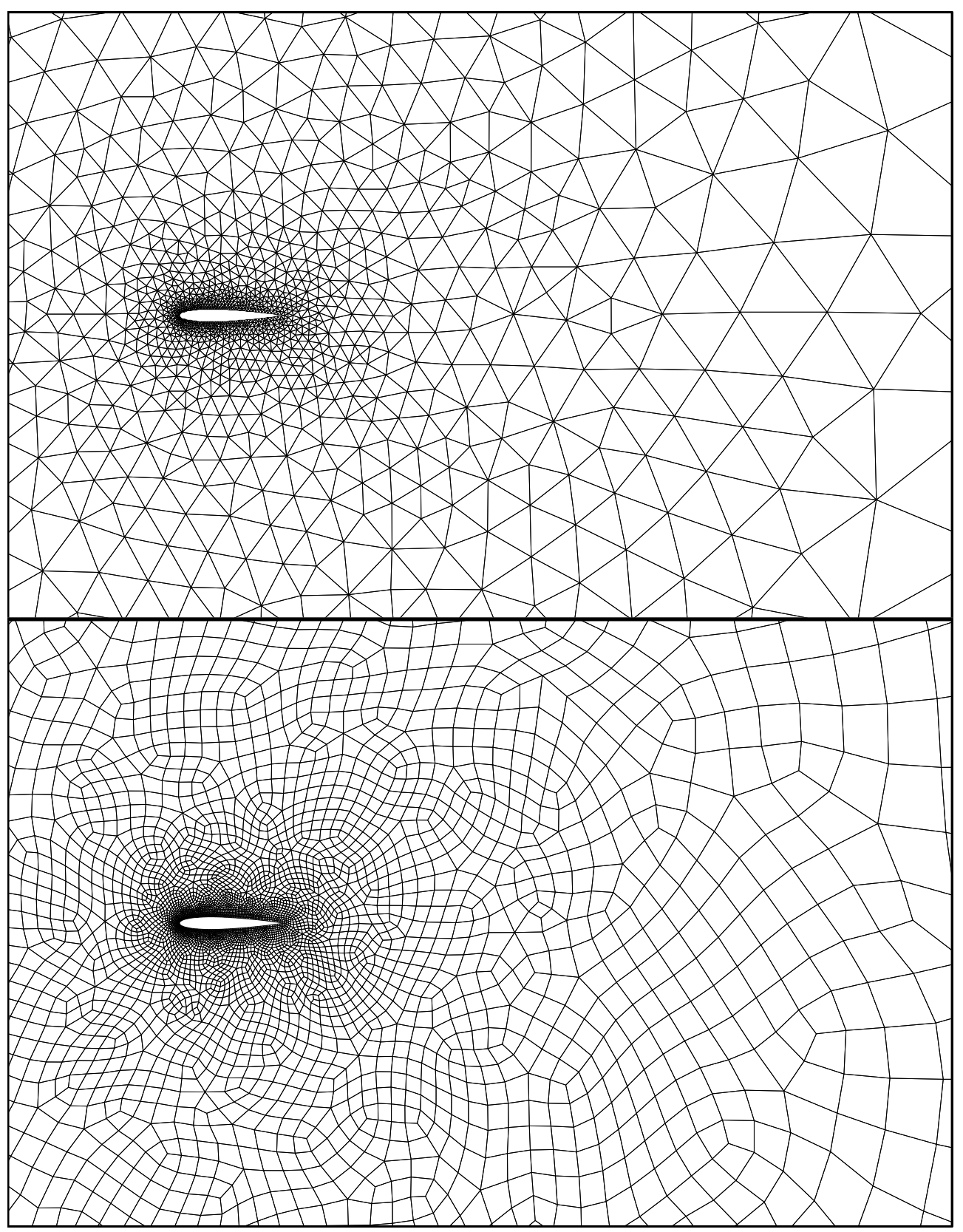

Figure 10: CFD meshes, initial triangular and quadrilateral isotropic meshes.

INRIA 


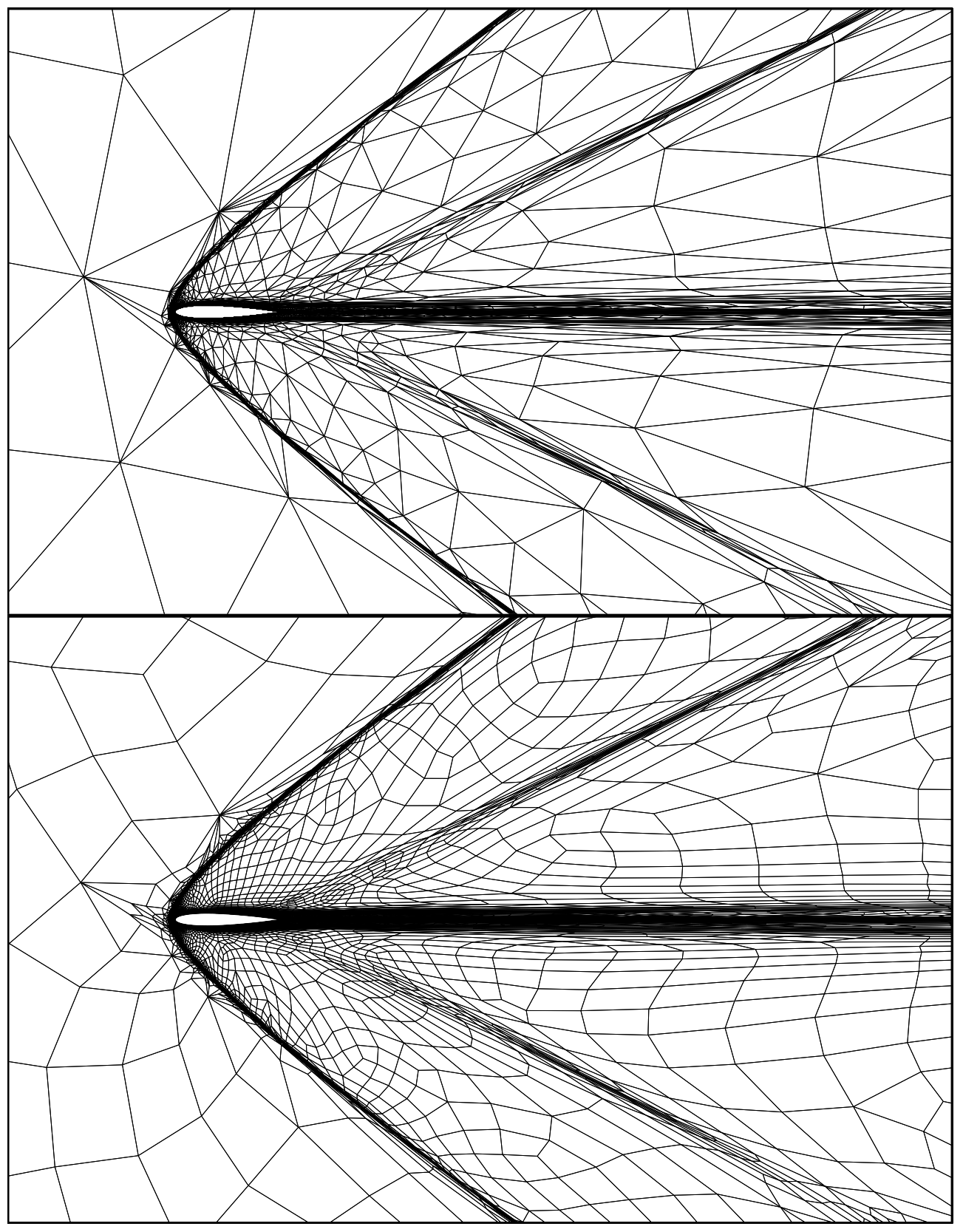

Figure 11: CFD meshes, final adapted triangular and quadrilateral anisotropic meshes.

RR $n^{\circ} 2960$ 


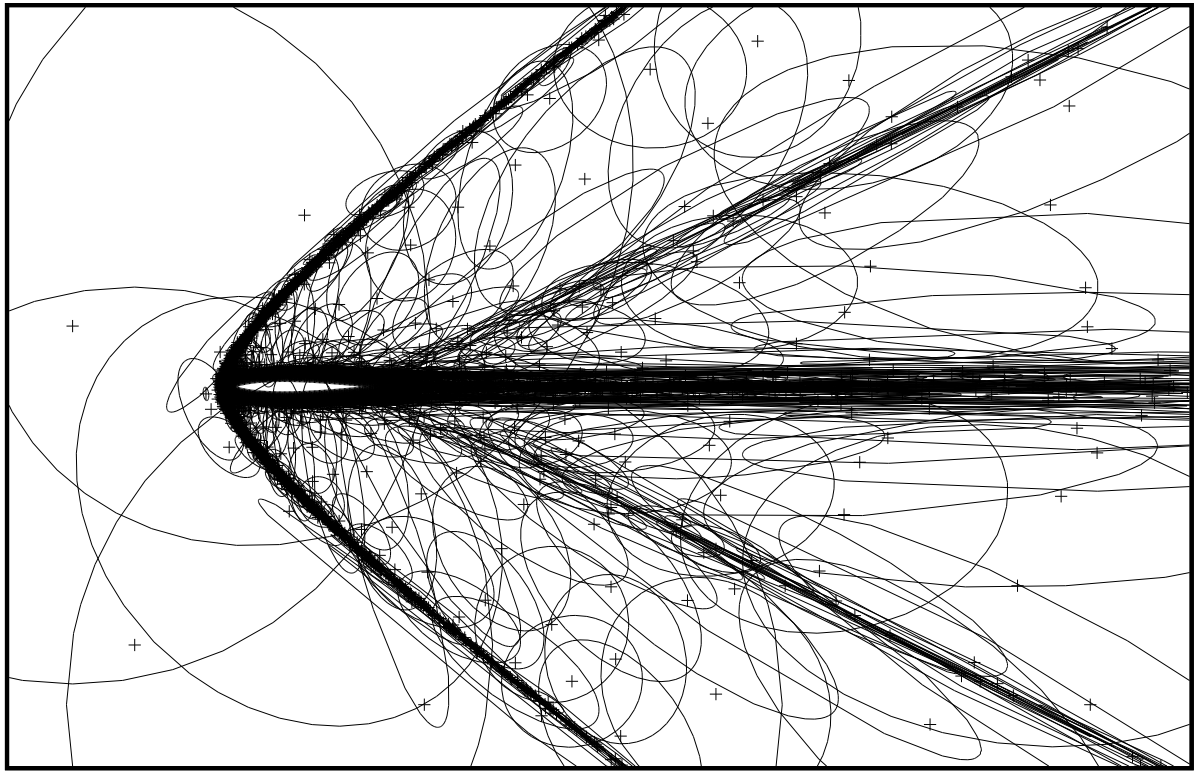

Figure 12: Metric map associated with the final adapted mesh.

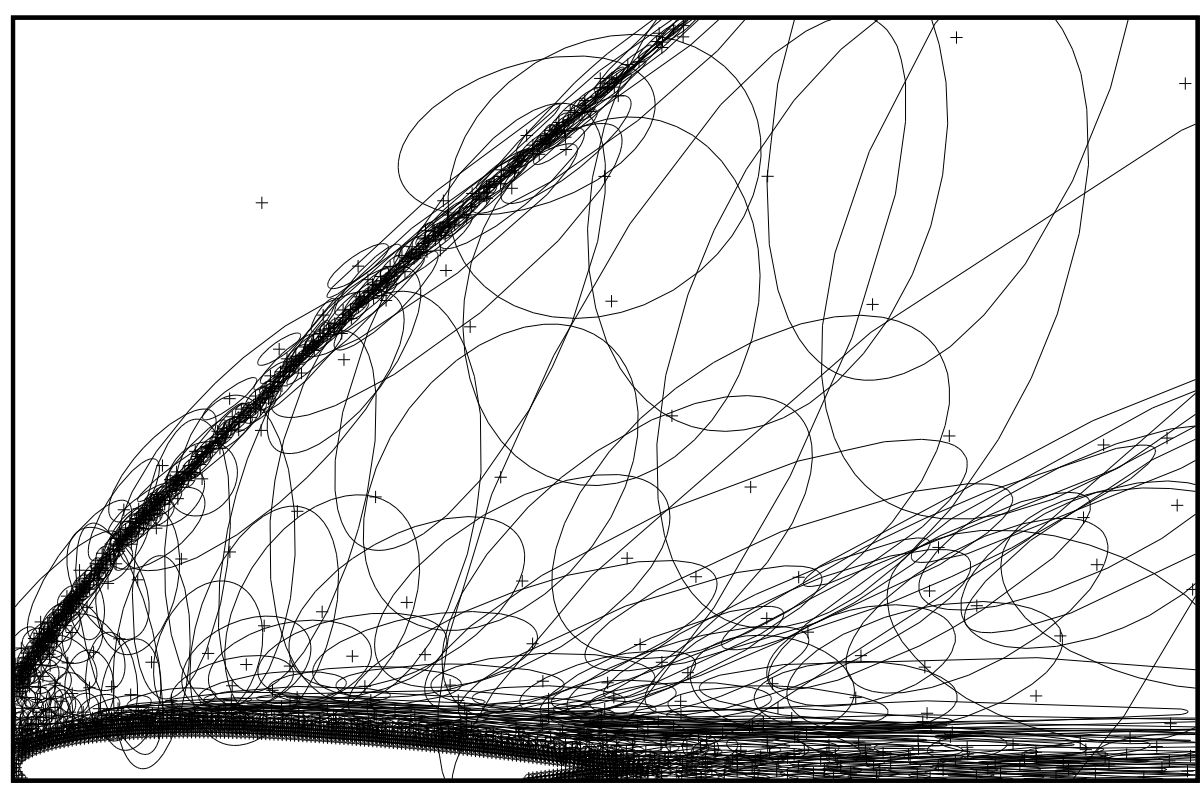

Figure 13: Metric map associated with the final adapted mesh (partial zoom).

INRIA 

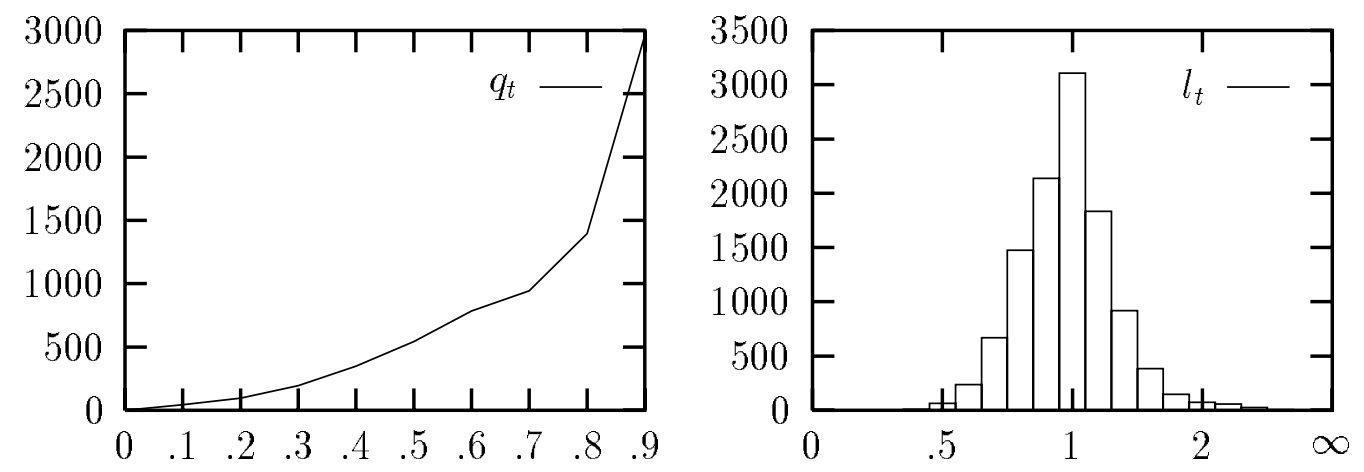

Figure 14: Triangular mesh quality. Figure 15: Normalized edge lengths.
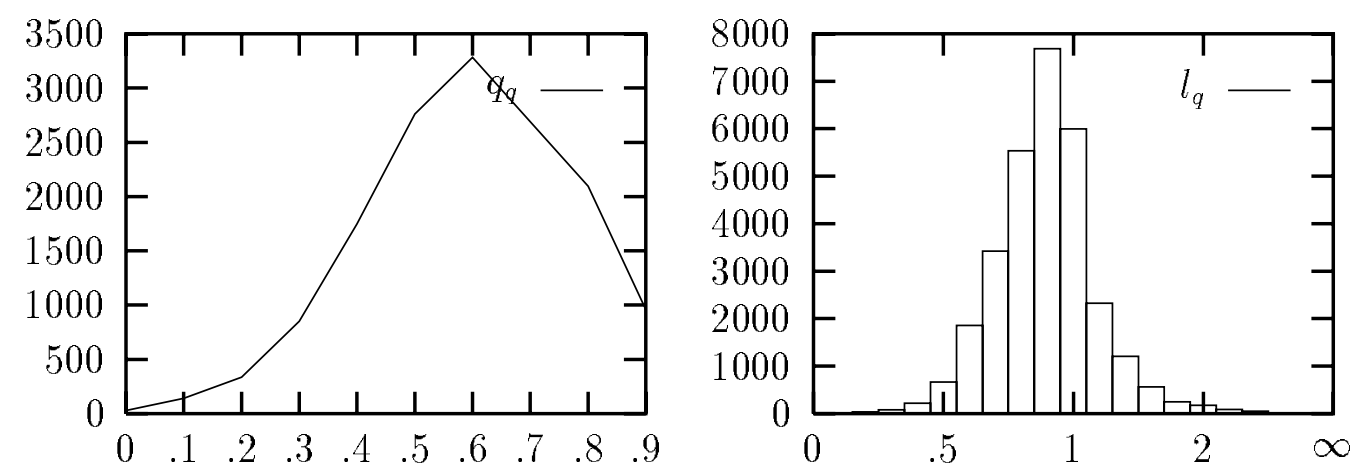

Figure 16: Quadrilateral mesh quality. Figure 17: Normalized edge lengths.

$\operatorname{RR} \mathrm{n}^{\circ} 2960$ 


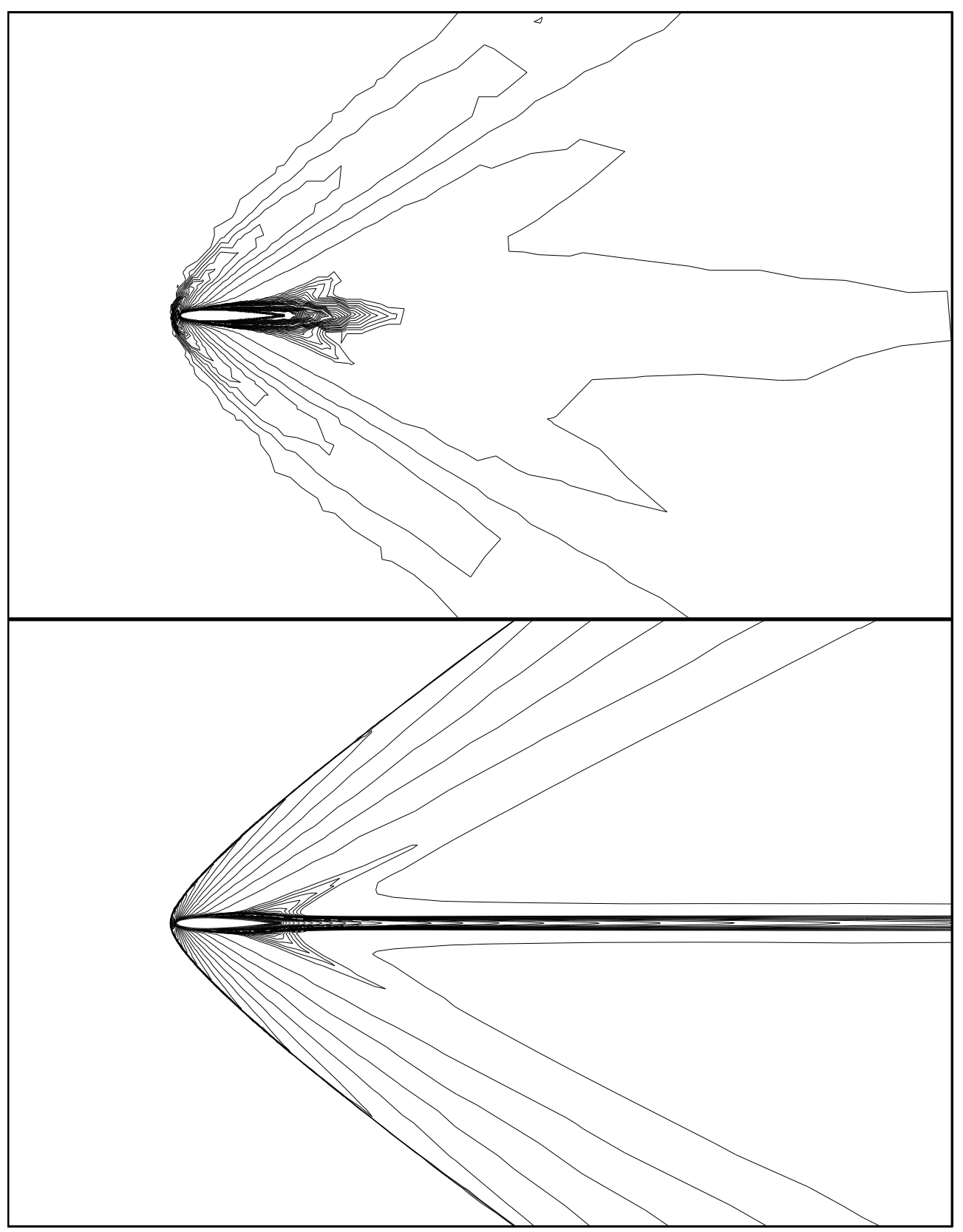

Figure 18: Iso-density contour lines of the solutions. 


\section{Conclusions}

In this paper, we have briefly reviewed an adaptive mesh generation method governed by a riemannian discrete metric map. In this context, we have introduced the concept of a unit mesh with respect to a riemannian structure. Then, we have proposed an automatic triangular to quadrilateral mesh conversion scheme, which takes the governing control space into account. Finally, we have introduced an optimal vertex smoothing procedure by means of normalized unit edge lengths. Several examples, in particular a CFD test, illustrated the efficiency of the proposed method.

A possible extension of the method consists in governing the merging process by adjacency using an advancing-front technique in order to obtain a pure quadrilateral mesh. This requires to generalize a topological classification of the configurations encountered in the merging of two fronts cases which eventually results in new vertices insertion. The proposed mesh generation scheme can be naturally extended to parametric surfaces triangular and quadrilateral mesh generation [7].

\section{References}

[1] C.G. Armstrong, D.J. Robinson, R.M. McKeag, T.S. Li, S.J. Bridgett And R.J. Donaghy, Applications of the medial axis transform in analysis modelling, Nafems, Proc. of the 5th Int. Conf. Reliability of FEM for Engng. Appl., pp. 415-426, Amsterdam, Netherlands, 10-12 May, 1995.

[2] M.J. Berger and A. Jameson, Automatic adaptive grid refinement for Euler equations, $A I A A J$. , vol 23, no 4, pp. 561-568, 1985.

[3] T.D. Blacker and M.B. Stephenson, Paving: A new approach to automated quadrilateral mesh generation, Int. J. Numer. Methods Eng., vol 32, pp. 811-847, 1991.

[4] H. Borouchaki, P.L. George, F. Hecht, P. Laug and E. Saltel, Delaunay mesh generation governed by metric specifications. Part I: Algorithms, to appear in Finite Elements in Analysis and Design.

$\operatorname{RR} n^{\circ} 2960$ 
[5] H. Borouchaki, P.L. George and B. Mohammadi, Delaunay mesh generation governed by metric specifications. Part II: Applications, to appear in Finite Elements in Analysis and Design.

[6] H. Borouchaki and P.L. George, Triangulation de Delaunay et métrique riemannienne. Applications aux maillages éléments finis, to appear in Revue européenne des éléments finis.

[7] H. Borouchaki, P.J. Frey et P.L. George, Maillage de surfaces paramétriques. Part III: Elements quadrangulaires, Rapport de Recherche INRIA, RR-2954, 1996.

[8] M.J. Castro-Diaz, F. Hecht and B. Mohammadi, New progress in anisotropic grid adaptation for inviscid and viscous flows simulations, Proc. of the 4th Int. meshing Roundtable, Albuquerque, NM, Oct. 16-17, 1995.

[9] B.P. Johnston, J.M. Sullivan and A. Kwasnik, Automatic conversion of triangular finite element meshes to quadrilateral elements, Int. J. Numer. Methods Eng., vol 31, pp. 67-84, 1991.

[10] C.K. LeE And S.H. Lo, A new scheme for the generation of a graded quadrilateral mesh, Comp. Struct., vol 52, pp. 847-857, 1994.

[11] R.W. Lewis, Y. Zheng and A.S. Usmani, Aspects of adaptive mesh generation based on domain decomposition and Delaunay triangulation., Finite Elements in Anal. and Design., vol 20, pp. 47-70, 1995.

[12] S.H. Lo, Generating quadrilateral elements on plane and over curved surfaces, Comp. Struct., vol 31, pp. 421-426, 1989.

[13] R. Lohner, Adaptive remeshing for transient problems, Comp. Methods Appl. Mech. Eng., vol 75, pp. 195-214, 1989.

[14] D.J. Mavriplis , Adaptive mesh generation for viscous flows using Delaunay triangulation, Jour. of Comput. Phys., vol 90, no 2, 1990.

[15] B. Mohammadi, Fluid Dynamics Computation with NSC2KE - a User guide, release 1.0, Technical Report INRIA, RT-164, 1994.

[16] J. Peraire, M. Vahdati, K. Morgan and O.C. Zienkiewicz, Adaptive remeshing for compressible flow computations, Jour. of Comput. Phys., vol 72, pp. 449-466, 1987. 
[17] E. Rank, M. Schweingruber and M. Sommer, Adaptive Mesh Generation., Commn. Numer. Methods Eng., vol 9, pp. 121-129, 1993.

[18] M.C. Rivara A 3-D Refinement Algorithm Suitable for Adaptive and MultiGrid Techniques, Commn. App. Numer. Methods, vol 8, pp. 281-290, 1992.

[19] J.A. Talbert and A.R. Parkinson, Development of an automatic twodimensional finite element mesh generator using quadrilateral elements and Bezier curve boundary definition, Int. J. Numer. Methods Eng., vol 29, pp. 1551$1567,1991$.

[20] D.F. Watson, Computing the n-dimensional Delaunay tessellation with application to Voronoï polytopes, Comput. J., vol. 24, pp. 167-172, 1981.

[21] N.P. Weatherill, M.J. Marchant, O. Hassan and D.L. Marcum, Grid adaptation using a distribution of sources applied to inviscid compressible flow simulations, Int. J. Numer. Methods Eng., vol 19, pp. 739-764, 1994.

[22] J.Z. Zhu, O.C. Zienkiewicz, E. Hinton And J. Wu, A new approach to the development of automatic quadrilateral mesh generation, Int. J. Numer. Methods Eng., vol 32, pp. 849-866, 1991.

$\operatorname{RR} \mathrm{n}^{\circ} 2960$ 
Unit'e de recherche INRIA Lorraine, Technopôle de Nancy-Brabois, Campus scientifique, 615 rue du Jardin Botanique, BP 101, 54600 VILLERS LÈS NANCY

Unit'e de recherche INRIA Rennes, Irisa, Campus universitaire de Beaulieu, 35042 RENNES Cedex Unit'e de recherche INRIA Rhône-Alpes, 655, avenue de l'Europe, 38330 MONTBONNOT ST MARTIN Unit'e de recherche INRIA Rocquencourt, Domaine de Voluceau, Rocquencourt, BP 105, 78153 LE CHESNAY Cedex Unit'e de recherche INRIA Sophia-Antipolis, 2004 route des Lucioles, BP 93, 06902 SOPHIA-ANTIPOLIS Cedex

Éditeur

INRIA, Domaine de Voluceau, Rocquencourt, BP 105, 78153 LE CHESNAY Cedex (France)

ISSN 0249-6399 